CERN-TH-2020-211

\title{
Higgs couplings: constraints and sensitivity on Supersymmetry
}

\author{
F. Mahmoudi, ${ }^{a, b, *}$ A. Arbey, ${ }^{a, b}$ M. Battaglia, ${ }^{c}$ A. Djouadi, ${ }^{d}$ M. Mühlleitner ${ }^{e}$ and \\ M. Spira ${ }^{f}$ \\ ${ }^{a}$ Université de Lyon, Université Claude Bernard Lyon 1, CNRS/IN2P3, \\ Institut de Physique des 2 Infinis de Lyon, UMR 5822, F-69622, Villeurbanne, France \\ ${ }^{b}$ Theoretical Physics Department, CERN, CH-1211 Geneva 23, Switzerland \\ ${ }^{c}$ University of California at Santa Cruz, Santa Cruz Institute of Particle Physics, CA 95064, USA \\ ${ }^{d}$ USMB-CNRS-LAPTh, F-74000 Annecy, France and NICPB, 10143 Tallinn, Estonia. \\ ${ }^{e}$ Institute for Theoretical Physics, Karlsruhe Institute of Technology, 76128 Karlsruhe, Germany \\ ${ }^{f}$ Paul Scherrer Institut, CH-5232 Villigen PSI, Switzerland \\ E-mail: nazila@cern.ch, alexandre.arbey@ens-lyon.fr, \\ marco.battaglia@ucsc.edu, abdelhak.djouadi@cern.ch, \\ milada.muehlleitner@kit.edu, michael.spira@psi.ch
}

The determination of the fundamental properties of the Higgs boson with sufficient accuracy has crucial implications for physics beyond the Standard Model. Here, we consider Supersymmetry and highlight the complementarity of the Higgs measurements and the superparticle searches at the $\mathrm{LHC}$, based on the 8 and $13 \mathrm{TeV}$ results, in probing its phenomenological minimal version, the pMSSM. In particular, we discuss the constraints that can be obtained on the supersymmetric parameters by the determination of the Higgs mass and couplings. In addition, we evaluate to which extent the high-luminosity LHC upgrade and a future electron-positron collider can help probing the pMSSM Higgs sector and reconstructing its underlying parameters.

40th International Conference on High Energy physics - ICHEP2020

July 28 - August 6, 2020

Prague, Czech Republic (virtual meeting)

\footnotetext{
${ }^{*}$ Speaker
} 


\section{Introduction}

The discovery of the Higgs boson in 2013 at the LHC [1] has initiated a broad program of studies of its properties, allowing for new tests of the Standard Model (SM) and also of New Physics (NP) beyond it. Here, we consider Supersymmetry (SUSY) which represents a good benchmark for $\mathrm{NP}$ and a rich laboratory for searches and studies. In contrast to the SM, where all the properties of the Higgs boson are uniquely determined once its mass is fixed, the couplings of the lightest Higgs boson in Supersymmetry, as well as its production and decay rates, can be significantly modified.

In the context of the phenomenological Minimal Supersymmetric Standard Model (pMSSM) with its 19 free parameters [2], we focus on the lightest Higgs boson and discuss the dependence of its mass and couplings on the relevant Higgs sector parameters, namely the pseudoscalar Higgs mass $M_{A}$ and the ratio of the Higgs vacuum expectation values $\tan \beta$. We also discuss the $\Delta_{b}$ corrections which come from higher order direct corrections due to SUSY particles in the loops. We will summarize the constraints derived from LHC searches and measurements and present the sensitivity of an electron-positron collider in the reconstruction of some pMSSM parameters. This work is based on Ref. [3] where more details and results will be given.

\section{Higgs couplings and SUSY corrections}

In the MSSM there are five Higgs states: two CP-even $h$ and $H$ boson where the former is considered to be the lightest with the measured mass of $125 \mathrm{GeV}$ [1], a CP-odd $A$ state and two charged Higgs bosons $H^{ \pm}$. The tree-level couplings of the neutral Higgs bosons to the up-quarks, down-quarks and charged leptons as well as vector bosons, normalized to the SM ones, are given in Table 1 as functions of $\beta$ and the mixing angle $\alpha$. The so-called decoupling limit, when the light Higgs couplings become equal to the SM Higgs ones, is reached for large $M_{A}$ such that $\alpha \approx \beta-\frac{\pi}{2}$.

\begin{tabular}{|c|c|c|c|}
\hline$\phi$ & $g_{\phi u \bar{u}}$ & $g_{\phi d \bar{d}}=g_{\phi \ell \bar{\ell}}$ & $g_{\Phi V V}$ \\
\hline$h$ & $\cos \alpha / \sin \beta \rightarrow 1$ & $-\sin \alpha / \cos \beta \rightarrow 1$ & $\sin (\beta-\alpha) \rightarrow 1$ \\
\hline$H$ & $\sin \alpha / \sin \beta \rightarrow-\cot \beta$ & $\cos \alpha / \cos \beta \rightarrow \tan \beta$ & $\cos (\beta-\alpha) \rightarrow 0$ \\
\hline$A$ & $\cot \beta$ & $\tan \beta$ & 0 \\
\hline
\end{tabular}

Table 1: MSSM tree level Higgs couplings relative to the SM couplings. In blue, shown are the values in the decoupling limit corresponding to $M_{A} \gg M_{Z}$.

As is well known, the MSSM Higgs sector which can be described at tree-level by only two parameters $\tan \beta$ and $M_{A}$, is affected by large radiative corrections. The important part of these corrections can be mapped into the lightest $h$ boson mass when it is fixed to the measured value $M_{h}=125 \mathrm{GeV}$ and one has for the mixing angle $\alpha$ for instance [4]

$$
\alpha \approx-\arctan \left(\frac{\left(M_{Z}^{2}+M_{A}^{2}\right) \cos \beta \sin \beta}{M_{Z}^{2} \cos ^{2} \beta+M_{A}^{2} \sin ^{2} \beta-M_{h}^{2}}\right) .
$$

There are also direct QCD and electroweak vertex corrections involving SUSY particles in the loops. In particular for the neutral Higgs states $\phi=h, H, A$ one has for a given fermion $f$ [5]

$$
g_{\phi f \bar{f}}^{\mathrm{eff}}=\frac{g_{\phi f \bar{f}}}{1+\Delta_{f}}\left(1-\frac{\Delta_{f}}{c_{\phi}}\right)
$$


with $c_{h}=\tan \alpha \tan \beta, c_{H}=-\tan \beta / \tan \alpha$ and $c_{A}=\tan ^{2} \beta$. These direct corrections $\Delta_{f}$ are particularly large for bottom quarks at high $\mu \tan \beta$ values. The main parts involve sbottom-gluino and stop-chargino loops and the expressions can be found in Ref. [5].

The LHC has provided measurements of the Higgs couplings and, in particular, of the couplings modifiers, i.e. effective Higgs couplings normalised to their SM values, $\kappa_{X}=g_{h X X}^{\mathrm{MSSM}} / g_{H_{\mathrm{SM} X X} \mathrm{SM}}^{\mathrm{S}}$. For this analysis, we use the experimental measurements of Ref. [6] which have been determined from the production and decay rates in many different channels measured at the LHC, and the projections for the coupling modifiers from Ref. [7].

In the following, we discuss the possibilities to distinguish the MSSM lightest $h$ state from the SM Higgs boson using measurements at the LHC and beyond. More specifically, we consider the phenomenological MSSM or pMSSM, which is the most general CP and R parity-conserving MSSM scenario which assumes Minimal Flavour Violation at the $\mathrm{TeV}$ scale and imposes the suppression of FCNCs at tree level. This leads to 19 free parameters: 10 sfermion masses $m_{\tilde{f}}, 3$ gaugino masses $M_{i}, 3$ trilinear couplings $A_{f}$ and 3 Higgs/higgsino parameters, $\mu, \tan \beta$ and $M_{A}$.

Following earlier work [8], we perform flat scans over the pMSSM 19 parameters varying all the masses between 0 and $6 \mathrm{TeV}$, the trilinear couplings between -15 and $15 \mathrm{TeV}$, and $\tan \beta$ between 1 and 60. The master program is based on SuperIso [9], we generate the MSSM spectra with SOFTSUSY [10] and Suspect [11] and compute the Higgs boson decay branching ratios and couplings using HDECAY [12]. We keep only points for which the lightest supersymmetric particle is the neutralino dark matter candidate and a light Higgs with $122<M_{h}<128 \mathrm{GeV}$. In most cases we include constraints from dark matter and $B$-meson physics.

\section{LHC constraints on the pMSSM}

We first study the effect of experimental selections from LHC high- $p_{T}$ and missing energy searches. For this purpose, we use many different SUSY and Higgs search channels in order to assess whether a pMSSM point is excluded or not by the data. Since the pMSSM is an unconstrained scenario, the constraints on the SUSY masses are less strict than in simplified scenarios. Figure 1 shows the fractions of pMSSM points compatible with LHC data as functions of the pseudoscalar Higgs, the gluino and the lightest neutralino and stop masses. As can be seen in the figure, in the pMSSM the gluinos can be as light as $1 \mathrm{TeV}$ and stops as light as $400 \mathrm{GeV}$. From heavy Higgs searches, only a few percent of the pMSSM points with $M_{A}<500 \mathrm{GeV}$ remain and less than $10 \%$ of those with $M_{A}<1 \mathrm{TeV}$. The surviving low $M_{A}$ points are more likely to have light Higgs couplings differing from the SM ones and the complementarity between the Higgs coupling measurements and heavy Higgs searches is thus particularly useful. Next, we consider the constraints from the light Higgs coupling measurements of Ref. [6]. In Figure 2, we present the couplings modifiers $\kappa_{i}$ for all valid pMSSM points and for those not excluded by the LHC Run 2 direct searches, compared to the ATLAS contours. As can be seen, most of the pMSSM points have coupling modifiers in agreement with the experimental results and $\kappa_{b}$ varies over a wide range. An important effect with significant impact on $\kappa_{b}$ comes from the $\Delta_{b}$ correction which, as discussed earlier, is proportional to $\mu \tan \beta$ and can in principle be of order unity, in particular in the large gluino mass limit or for a large trilinear stop coupling $A_{t}$. The effect of $\Delta_{b}$ on $\mu_{b b}$ is illustrated in Figure 3, where $\mu_{b b}$ is the ratio of the branching ratio of the $h \rightarrow b \bar{b}$ decay over its SM value. However, as shown in 

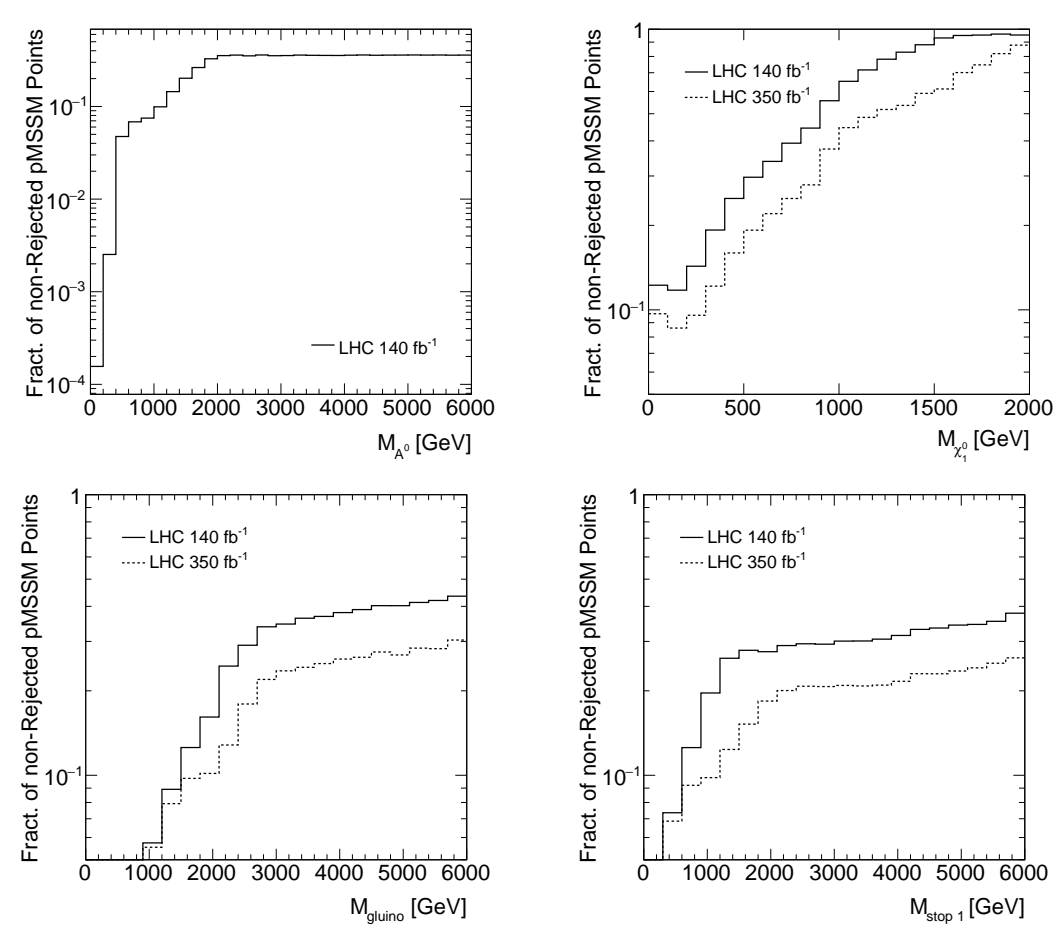

Figure 1: Fraction of pMSSM points compatible with LHC high- $p_{T}$ searches, with the current $140 \mathrm{fb}^{-1}$ data and extrapolated to $300 \mathrm{fb}^{-1}$ as a function of the pseudoscalar Higgs, gluino, neutralino and stop masses.
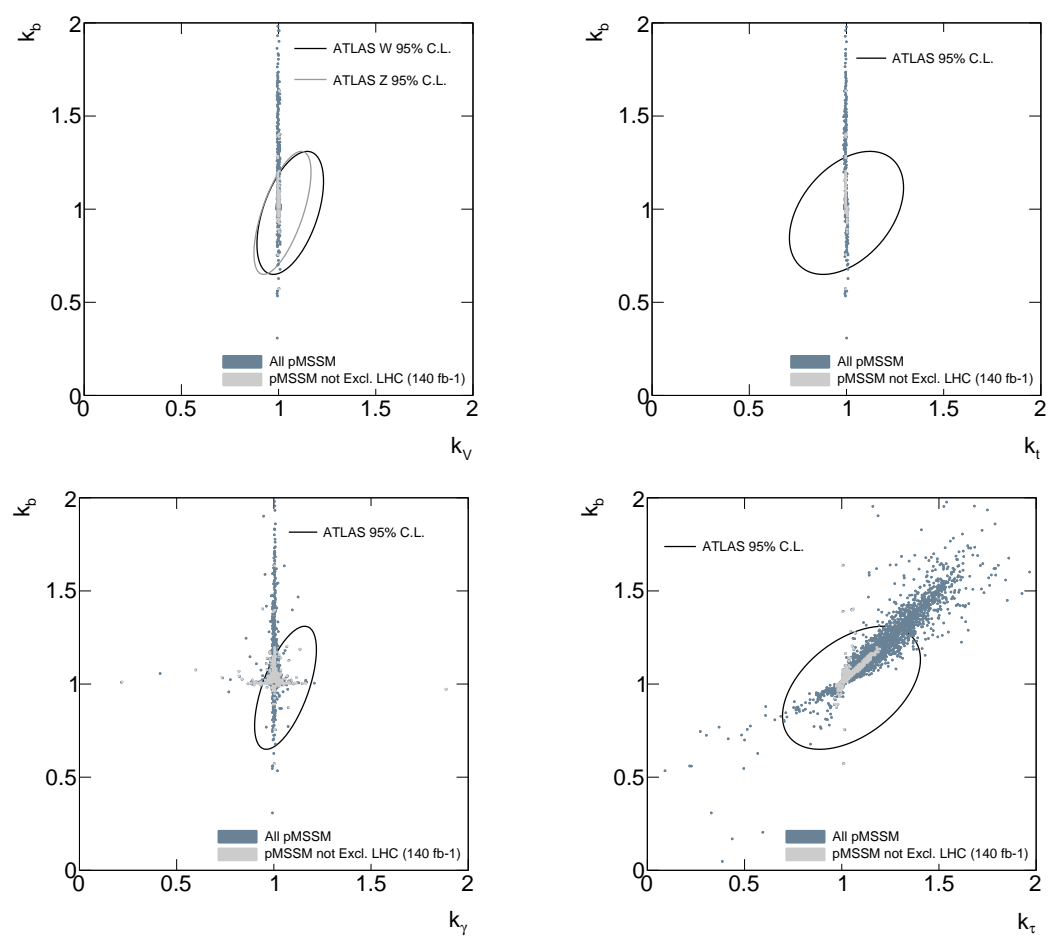

Figure 2: pMSSM valid points (dark colour) and points not excluded by LHC searches (light colour) in the $\kappa_{b}$ vs. $\kappa_{V}$ (upper left), $\kappa_{t}$ (upper right), $\kappa_{\gamma}$ (lower left) and $\kappa_{\tau}$ (lower right) parameter planes. Superimposed are the experimental contours at $95 \%$ C.L. of ATLAS [6]. 

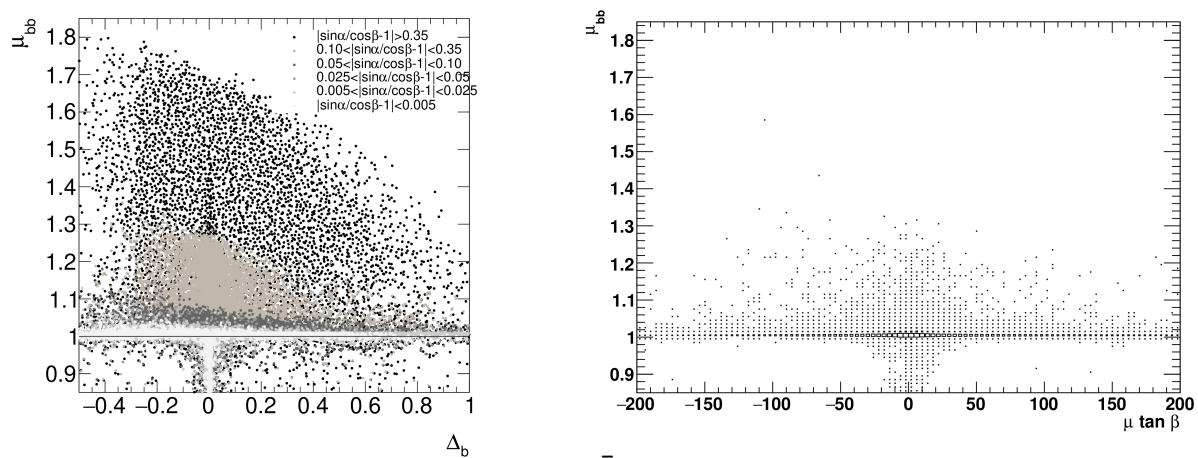

Figure 3: (Left) Ratio of branching ratio of $h \rightarrow b \bar{b}$ normalised to its SM value vs. $\Delta_{b}$ for different values of $|\sin \alpha / \sin \beta|$. (Right) In the decoupling limit with $M_{A}>250 \mathrm{GeV}$, ratio of branching ratio of $h \rightarrow b \bar{b}$ normalised to its SM value vs. $\mu \tan \beta$, where the size of the squares reflects the density of pMSSM points.

the right plot, there exists only a mild correlation between $\mu \tan \beta$ and $\mu_{b b}$, and the small shifts are dominated by deviations of the mixing angle $\alpha$ from the SM limit. This is due to a suppression of the $\Delta_{b}$ corrections in the region of $M_{A}$ still compatible with the experimental constraints. More generally we found that within our pMSSM scan, the $M_{A}$ values at which more than $95 \%$ of the accepted pMSSM points are excluded by the Higgs coupling measurements at 95\% C.L. are below $275 \mathrm{GeV}$ for the present Run 2 ATLAS data. When considering in addition direct searches, this limit is pushed to $325 \mathrm{GeV}$. With the HL-LHC run, one can expect these values to reach $525 \mathrm{GeV}$ and $725 \mathrm{GeV}$, respectively.

The fraction of pMSSM points that can be probed by the Higgs coupling measurements at the LHC remains small, in spite of the increasing accuracy of the Higgs measurements. Beyond LHC, percent or sub-percent accuracy at a $e^{+} e^{-}$Higgs factory makes this fraction to reach almost $10 \%$.

\section{Determination of SUSY parameters at an electron-positron collider}

We then examine the possibility of reconstructing the MSSM parameters by measuring more precisely the Higgs couplings at a future Higgs factory. As an illustration, we discuss the optimal case of a linear $e^{+} e^{-}$collider with a $1 \mathrm{TeV}$ energy in the center of mass, and study benchmark points which are currently compatible with Higgs and SUSY direct searches at the LHC, with masses as low as $434 \mathrm{GeV}$ and at low and intermediate $\tan \beta$ between 3 and 10 . The sparticles do not have a significant effect on the branching ratios and the main sensitivity is indeed on $M_{A}$.

In Figure 4, we show the most probable $M_{A}$ values of the probability density functions obtained by weighing each accepted pMSSM point with its $\chi^{2}$ probability to have Higgs couplings compatible with those predicted for a given pMSSM benchmark point assuming the anticipated accuracy [7], as a function of the expected mass for all the benchmark points. This shows that the reconstruction can be very precise for a light $A$ state and the uncertainties increase with $M_{A}$, but that it is still possible to reconstruct a mass of $1 \mathrm{TeV}$.

\section{Summary}

We have summarized the work of Ref. [3] where we study the complementarity between direct searches and Higgs coupling measurements at the LHC to probe the parameter space of the pMSSM 


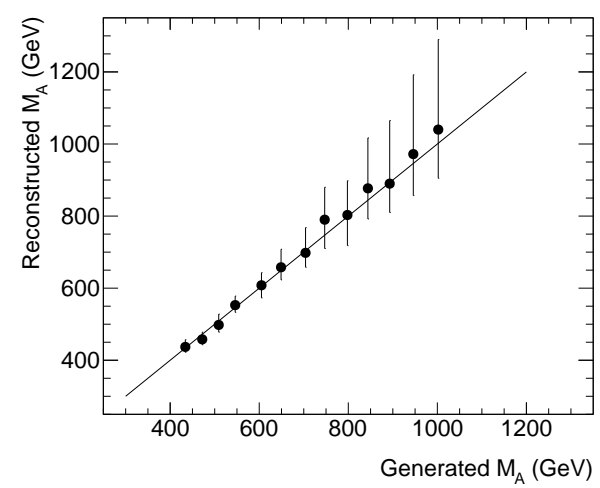

\begin{tabular}{|c|c|c|c|}
\hline$M_{A}(\mathrm{GeV})$ & $\tan \beta$ & $\mu$ & $M_{\chi_{1}^{0}}$ \\
\hline \hline 434.4 & 5.58 & -549.3 & 562.1 \\
472.4 & 6.62 & 1993.7 & 314.8 \\
509.8 & 5.48 & -181.9 & 184.7 \\
546.9 & 5.55 & -50.5 & 49.9 \\
605.7 & 6.31 & 369.1 & 380.1 \\
649.5 & 3.15 & 1722.6 & 108.3 \\
704.4 & 5.24 & 480.5 & 170.6 \\
747.0 & 4.51 & -3596.1 & 1072.0 \\
798.4 & 5.75 & -3301.7 & 1329.4 \\
893.2 & 7.14 & -367.9 & 379.5 \\
1001.9 & 5.39 & 715.9 & 732.0 \\
\hline
\end{tabular}

Figure 4: Most probable values of the pseudoscalar $A$ mass at a $1 \mathrm{TeV} e^{+} e^{-}$collider to have Higgs couplings compatible with the measurements, for 11 pMSSM benchmark points. Details on the properties of the original pMSSM benchmark points are provided in the table.

with its 19 free parameters. We have shown that the variations of the MSSM light Higgs couplings allowed by the current direct searches are mostly within the 95\% C.L. of the ATLAS Higgs coupling measurements. We have also demonstrated that the sensitivity of Higgs coupling measurements on the MSSM parameters is through $M_{A}$ and $\tan \beta$, which are also constrained through direct LHC searches. There is still room for improving these constraints or discovering new effects, both at the high-luminosity LHC and at a future $e^{+} e^{-}$collider acting as a Higgs factory, and we have seen that a $1 \mathrm{TeV} e^{+} e^{-}$collider will have the capability to reconstruct $M_{A}$ up to $1 \mathrm{TeV}$.

\section{References}

[1] G. Aad et al. [ATLAS Collaboration], Phys. Lett. B716 (2012) 1; S. Chatrchyan et al. [CMS Collaboration], Phys. Lett. B716 (2012) 30.

[2] A. Djouadi et al. [MSSM Working Group], arXiv:hep-ph/9901246.

[3] A. Arbey, M. Battaglia, A. Djouadi, F. Mahmoudi, M. Mühlleitner and M. Spira, to appear.

[4] A. Djouadi et al., Eur. Phys. J. C73 (2013) 2650; JHEP 06 (2015) 168.

[5] For a recent discussion see M. Ghezzi et al., arXiv:1711.02555.

[6] G. Aad et al. [ATLAS Collaboration], Phys. Rev. D 101 (2020) no.1, 012002.

[7] J. de Blas et al., JHEP 01 (2020), 139.

[8] A. Arbey et al., Eur.Phys.J.C72 (2012) 1847; JHEP09 (2012) 107; Phys.Lett. B708 (2012) 162; Phys.Lett. B720 (2013) 153; Phys.Rev. D89 (2014) 077701; Phys.Rev. D94 (2016) 055015.

[9] F. Mahmoudi, Comput. Phys. Commun. 180 (2009) 1579; A. Arbey and F. Mahmoudi, Comput. Phys. Commun. 181 (2010) 1277; Comput. Phys. Commun. 239 (2019) 238.

[10] B. C. Allanach, Comput. Phys. Commun. 143 (2002) 305.

[11] A. Djouadi, J.L. Kneur and G. Moultaka, Comput. Phys. Commun. 176 (2007) 426.

[12] A. Djouadi, J. Kalinowski and M. Spira, Comput. Phys. Commun. 108 (1998) 56; A. Djouadi, J. Kalinowski, M. Mühlleitner and M. Spira, Comput. Phys. Commun. 238 (2019) 214. 